\title{
O ensino de ética médica como parte da graduação em medicina: Revisão sistemática de literatura
}

\section{The teaching of medical ethics as part of undergraduate studies in medicine: Systematic literature review}

\section{La enseñanza de ética médica como parte de la licenciatura en medicina: Revisión sistemática de literatura}

\section{Thiago Pontes de Oliveira César}

Como citar esse artigo. César, TPO.

O ensino de ética médica como parte da graduação em medicina: Revisão sistemática de literatura. Revista PróUniverSUS. 2020 Jul./Dez.; 11 (2): $27-$ 32 .

\section{Resumo}

O objetivo deste estudo foi verificar, por meio de uma revisão sistemática, com a ética médica no ensino de graduação de medicina é abordada na literatura. Trata-se de uma revisão sistemática de estudos publicados em português, de 2013 a 2020, disponíveis nas bases de dados da Biblioteca Virtual em Saúde (BVS), SciELO, Lilacs e Medline, utilizando os descritores: Ética, Educação Médica e Estudantes de Medicina. A partir da leitura e análise crítica de 16 artigos pré-selecionados, 12 foram considerados de maior relevância para a realização da pesquisa. Verifica-se que os assuntos discutidos na literatura são diversificados, abrangendo um amplo campo de questões que envolvem métodos alternativos de ensino da ética na graduação, análise de problemas morais, como os que envolvem o sigilo médico na relação médico-paciente e percepção dos estudantes sobre a disciplina e/ou ensino de ética médica. Estudos mostram que na percepção dos estudantes de medicina existe uma desvalorização do ensino de ética médica na graduação, bem como a dissociação entre teoria e prática. Ficou evidente que o conhecimento dos estudantes sobre o sigilo na relação médico-paciente ainda é deficiente, mesmo para alunos em níveis avançados da graduação. Havendo consenso de que a conduta ética precisa começar a ser desenvolvida ainda na formação inicial do estudante, a fim de se construir um agir profissional eticamente correto. Conclui-se que existem deficiências no ensino de ética médica, mas essas podem ser superadas quando houver a valorização da disciplina nos cursos de graduação de medicina e, sobretudo, nas universidades brasileiras.

Palavras-chave: Ética, Educação Médica, Estudantes de Medicina.

\begin{abstract}
The objective of this study was to verify, through a systematic review, with medical ethics in undergraduate teaching of medicine is addressed in the literature. This is a systematic review of studies published in Portuguese, from 2013 to 2020, available in the databases of the Virtual Health Library (VHL), Scielo, Lilacs and Medline, using the descriptors: Ethics, Medical Education and Medical Students. From the reading and critical analysis of 16 pre-selected articles, 12 were considered of greater relevance to the research. It is found that the subjects discussed in the literature are diverse, covering a wide field of issues involving alternative teaching methods of ethics in undergraduate, analysis of moral problems, such as those involving medical secrecy in the medical relationship-patient and students' perception of the discipline and/or teaching of medical ethics. Studies show that in the perception of medical students there is a devaluation of the teaching of medical ethics in undergraduate, as well as the dissociation between theory and practice. It was evident that students' knowledge of confidentiality in the doctor-patient relationship is still deficient, even for students at advanced levels of graduation. There is a consensus that ethical conduct must begin to be developed in the initial formation of the student, in order to build an ethically correct professional action. It is concluded that there are deficiencies in the teaching of medical ethics, but these can be overcome when there is an appreciation of the discipline in undergraduate medical courses and, above all, in Brazilian universities.
\end{abstract}

Keywords: Ethics, Medical Education, Medical Students. 


\section{Resumen}

El objetivo de este estudio fue verificar, por medio de una revisión sistemática, con la ética médica en la enseñanza de graduación de medicina es abordada en la literatura. Se trata de una revisión sistemática de estudios publicados en portugués, de 2013 a 2020, disponibles en las bases de datos de la Biblioteca Virtual en Salud (BVS), Scielo, Lilacs y Medline, utilizando los descriptores: Ética, Educación Médica y Estudiantes de Medicina. A partir de la lectura y análisis crítico de 16 artículos preseleccionados, 12 fueron considerados de mayor relevancia para la realización de la investigación. Resulta que los temas discutidos en la literatura son diversos, abarcando un amplio campo de cuestiones que involucran métodos alternativos de enseñanza de la ética en la graduación, análisis de problemas morales, como los que implican el secreto médico en la relación médico-paciente y percepción de los estudiantes sobre la disciplina y/o enseñanza de ética médica. Estudios muestran que en la percepción de los estudiantes de medicina existe una desvalorización de la enseñanza de ética médica en la graduación, así como la disociación entre teoría y práctica. Quedó claro que el conocimiento de los estudiantes sobre el secreto en la relación médico-paciente sigue siendo deficiente, incluso para alumnos en niveles avanzados de la graduación. Habiendo consenso de que la conducta ética necesita comenzar a ser desarrollada aún en la formación inicial del estudiante, a fin de construir un actuar profesional éticamente correcto. Se concluye que existen deficiencias en la enseñanza de ética médica, pero éstas pueden ser superadas cuando exista la valorización de la disciplina en los cursos de graduación de medicina y, sobre todo, en las universidades brasileñas.

Palabras clave: Ética, Educación Médica, Estudiantes de Medicina.

\section{Introdução}

Na graduação de medicina, o ensino da ética médica, da bioética e das humanidades são elementos essenciais para a formação dos futuros profissionais médicos, pois é na graduação que o estudante constrói as bases de sua futura relação médico-paciente ${ }^{1}$.

Os primeiros relatos de princípios éticos relativos à profissão médica são atribuídos a Hipócrates, que viveu há quatro séculos antes da era cristã ${ }^{2}$. Como parte da filosofia, a ética se caracteriza pelo saber racional, fundamentado em três pilares principais, consciência, autonomia e coerência ${ }^{3}$. O conceito de ética médica é amplo, ultrapassa a ideia de um conjunto de normas, implica em reflexão e decisão, e precisa ser percebido contextualmente, pois sofre interferência social e da relação do homem com o ambiente 4 .

Para Camargo et al. ${ }^{5}$, a ética implica um juízo crítico sobre valores, sendo, portanto, o desenvolvimento do senso crítico elemento essencial na formação médica, pois na prática profissional não basta ser capaz de distinguir o certo do errado. Para isso, é indispensável que o ensino da medicina extrapole a capacitação técnico-científica, de forma a preparar também o futuro médico para lidar com os dilemas, conflitos e pluralidade de opiniões na sua área de atuação ${ }^{6}$.

O desenvolvimento tecnológico na área da saúde, acompanhado pelo desenvolvimento farmacêutico e uso dessas novas tecnologias no cotidiano profissional têm requerido cada vez mais a discussão de questões éticas na formação profissional do médico, uma vez que essas inovações fizeram surgir outros valores no âmbito das relações interpessoais, colocando em questão novos conflitos ${ }^{7}$.

As Diretrizes Curriculares Nacionais do Curso de Graduação em Medicina recomendam que assuntos relacionados à bioética e à ética médica sejam incluídos na formação dos graduandos de medicina, sendo de responsabilidade das instituições de ensino superior
(IES) fazer com isso aconteça $a^{4,7}$.

Devendo envolver todos os docentes e não exclusivamente aqueles responsáveis pela disciplina de ética médica, pois cada um precisa trabalhar o conteúdo específico de sua disciplina de maneira que contemple discussões em torno das orientações, preceitos e princípios éticos atinentes à formação profissional, e isso contribuirá para tornar o aluno atento ao comportamento ético da profissão ${ }^{2}$.

Diante disso, a questão que norteia esta revisão é: Como a literatura aborda o ensino de ética médica entre os graduandos de medicina? Sendo assim, o objetivo deste estudo foi verificar, por meio de uma revisão sistemática, com a ética médica no ensino de graduação de medicina é abordada na literatura.

\section{Materiais e Métodos}

Foram acessadas no dia 07 de agosto de 2020 as bases de dados da Biblioteca Virtual em Saúde (BVS), Scielo, Medline e Lilacs, usando os descritores: Ética, Educação Médica e Estudantes de Medicina.

Figura 1. Fluxograma das publicações conforme as bases de dados, Scielo, Medline, Lilacs e BVS.

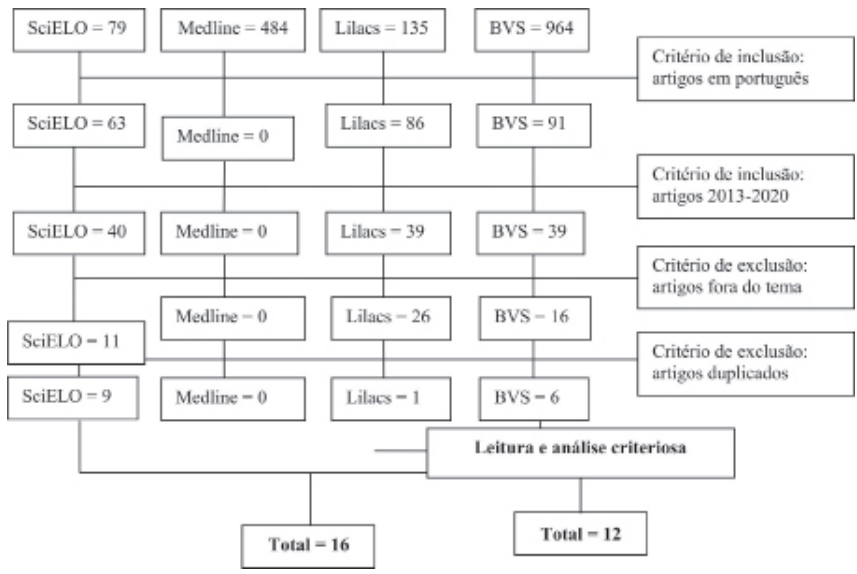

Fonte: Produção dos autores. 
Sendo estipulados os seguintes critérios de inclusão: artigos disponíveis na íntegra, publicados de 2013 a 2020 em português. Quanto ao critério de exclusão considerou-se o seguinte: resumos de artigos; artigos em outros idiomas que não fosse o português e artigos cujos temas não fossem considerados relevantes para esta revisão.

Os artigos foram analisados e categorizados com vista ao delineamento dos estudos, observando-se: ano de publicação, autor, objetivos, metodologia, resultados e conclusões.

Quadro 1 - Distribuição dos estudos conforme o ano de publicação, título, autoria, objetivos e desenho metodológico.

\begin{tabular}{|c|c|c|c|c|}
\hline ANO & AUTOR & TituLo & OBJETIVOS & METODO \\
\hline 2013 & Savaris et all'". & $\begin{array}{l}\text { Julgamento simulado } \\
\text { como estratégia de } \\
\text { ensino da ética módica. }\end{array}$ & $\begin{array}{l}\text { ldentificar a percepçầ dus alunus subere } \\
\text { a importância do julgansento simulado } \\
\text { cơno estratégia de ensino da ética } \\
\text { médica cm uma escola de medicina. }\end{array}$ & Estudo descritivo e transversal \\
\hline 2014 & Camargo et als. & $\begin{array}{l}\text { Ética c Bioctica: o que } \\
\text { cs alunos do sexto ano } \\
\text { módico têm a dizer. }\end{array}$ & 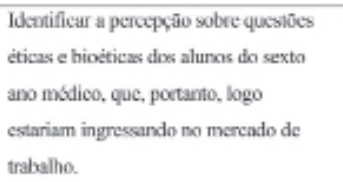 & $\begin{array}{l}\text { Estudo qualitativo de caráter } \\
\text { descritivo. }\end{array}$ \\
\hline 2014 & Yamaki ct al2. & 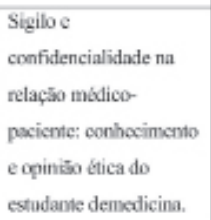 & $\begin{array}{l}\text { Avaliar o conhecimento e a opinișo } \\
\text { ética dos estudartes de medicina da } \\
\text { Universidade do Essado do Pará, } \\
\text { baseado no princípio do sigilo mádico. }\end{array}$ & $\begin{array}{l}\text { Estude prospectivo e } \\
\text { transversal }\end{array}$ \\
\hline 2014 & Lisboa ef all', & $\begin{array}{l}\text { Código de ética do } \\
\text { estudtanle de maticina: } \\
\text { uma análise qualitativan }\end{array}$ & $\begin{array}{l}\text { Companar os códigos de ética do } \\
\text { estulante de madicina existentes no } \\
\text { Bensil }\end{array}$ & $\begin{array}{l}\text { Estudo qualitativo de caníter } \\
\text { exp̨luratónio e descritiva. }\end{array}$ \\
\hline 2014 & Godoy et al ${ }^{11 .}$. & $\begin{array}{l}\text { Avaliaç̧io do } \\
\text { Conhecimentis da Ética } \\
\text { Médica dos } \\
\text { Gruàuandas de } \\
\text { Medicina. }\end{array}$ & $\begin{array}{l}\text { Mensurar o conbocimento referente ao } \\
\text { uema Ética Médica alquirido pelos } \\
\text { alunos do curso de Medicina da } \\
\text { Fasuldade de Medicina de Sâo Joś do } \\
\text { Rio Preto (Famerp). }\end{array}$ & Estudo transversal descritivo \\
\hline 2017 & Balbi et al: & $\begin{array}{l}\text { A Litcratura como } \\
\text { estratégia para } \\
\text { reflexỏes sobre } \\
\text { humanismo e ética no } \\
\text { curso médico: um } \\
\text { estudo qualitativo }\end{array}$ & 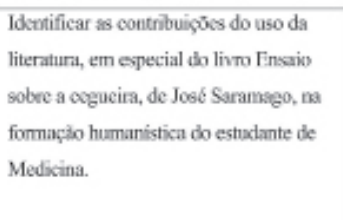 & $\begin{array}{l}\text { Estudo descritivo, de análise } \\
\text { qualitativa. }\end{array}$ \\
\hline 2017 & Menezes et al? & $\begin{array}{l}\text { Conflitos éticos } \\
\text { vivenciados por } \\
\text { estudanies de medicina. }\end{array}$ & $\begin{array}{l}\text { Identificar e analisar a prevalència de } \\
\text { conflitos éticos vivenciados por } \\
\text { estudantes de Medicina. }\end{array}$ & $\begin{array}{l}\text { Pesquisa com delineamento } \\
\text { transversal } e \text { analitico. }\end{array}$ \\
\hline 2019 & Menezes et al. & $\begin{array}{l}\text { Peroeppobs sobreo } \\
\text { ensino de ética na } \\
\text { medicina: estudo } \\
\text { qualitativo. }\end{array}$ & $\begin{array}{l}\text { Identificar peroeppós dos estudantes de } \\
\text { medicina quanio av ensino de ética na } \\
\text { graduacalo. }\end{array}$ & $\begin{array}{l}\text { Pesquisa empirica, com } \\
\text { aburdagem qualitativa. }\end{array}$ \\
\hline 2019 & Teixeira el al ${ }^{4}$ & $\begin{array}{l}\text { Biocética e discentes de } \\
\text { medicina: transicso do } \\
\text { métrodo de ensino. }\end{array}$ & $\begin{array}{l}\text { Relacionar o conhecimento de alunos de } \\
\text { medicina de duas modalidades de } \\
\text { ensino distintas (tradicional e ativa) } \\
\text { sobere o Cúdigo de Ética Médica. }\end{array}$ & $\begin{array}{l}\text { Estudo transversal, descritivo, } \\
\text { observacional e de análise } \\
\text { quantitativa. }\end{array}$ \\
\hline 2019 & Graças et al ${ }^{3}$. & $\begin{array}{l}\text { Conhecimento sobre } \\
\text { ética médica e } \\
\text { resoluçäo de conflitos } \\
\text { na graduaças. }\end{array}$ & $\begin{array}{l}\text { Avaliar a percospło do graduando cm } \\
\text { medicina spobre a importaincia do ensino } \\
\text { da ćtica modica e mensurar scu } \\
\text { conhecimento sobre a temática em } \\
\text { universidade publica do Nordeste } \\
\text { brasileim. }\end{array}$ & $\begin{array}{l}\text { Estudo quantitativo } \\
\text { transversal descritivo. }\end{array}$ \\
\hline \multirow[t]{2}{*}{2020} & Lima et al. ${ }^{12}$ & $\begin{array}{l}\text { Avaliaçio do } \\
\text { conhecimento de }\end{array}$ & $\begin{array}{l}\text { Avaliar o conhecimento dos estudantes } \\
\text { de mudicina de uma universidade }\end{array}$ & $\begin{array}{l}\text { Pesquisa empirica, com } \\
\text { abordagem qualitativa. }\end{array}$ \\
\hline & & $\begin{array}{l}\text { estudanles de medicina } \\
\text { sobre sigilo médico }\end{array}$ & $\begin{array}{l}\text { privada de Salvador/BA sobre o sigilo } \\
\text { na relaçâo médico-prciente. }\end{array}$ & \\
\hline 2020 & Barbosa et al? & $\begin{array}{l}\text { Conhecimento em ética } \\
\text { médica entre } \\
\text { estudanies de medicina } \\
\text { de Salvadur }\end{array}$ & $\begin{array}{l}\text { Avaliar o nivel de conhecimento em } \\
\text { ética médica de estudanles de medicina } \\
\text { de universidade privada de } \\
\text { Salvador/BA. }\end{array}$ & $\begin{array}{l}\text { Estudo observacional, do tipo } \\
\text { transversal, }\end{array}$ \\
\hline
\end{tabular}

Fonte: Dados do estudo, 2020. 


\section{Resultados e Discussão}

A partir da leitura e análise crítica de 16 artigos pré-selecionados que melhor atendiam ao tema de pesquisa, 12 foram considerados de maior relevância para compor este estudo. O Quadro 1 elenca os estudos conforme o ano de publicação, título, autoria, objetivos e desenho metodológico. O Quadro 2 apresenta os resultados e conclusões das publicações pesquisadas.

Verifica-se que os assuntos discutidos na literatura são diversificados, abrangendo um amplo campo de questões que envolvem métodos alternativos de ensino da ética na graduação, análise de problemas morais, como os que envolvem o sigilo médico na relação médico-paciente e percepção dos estudantes sobre a

Quadro 1 . Distribuição dos artigos conforme o ano de publicação, autoria, resultados e conclusões.

\begin{tabular}{|c|c|c|c|}
\hline ANO & AUTOR & RESULTADOS & CONCLUSÁO \\
\hline 2013 & Savaris et al ${ }^{10}$. & $\begin{array}{l}\text { Os resultados deste estudo mostraram que } \\
\text { os cstudantes de medicina cntrevistados, cm } \\
\text { grau de escalonamento variável, entendem a } \\
\text { importấncia da utilização do julgamento } \\
\text { simulado como estratégia de ensino da ética } \\
\text { médica e para a formação profissional do } \\
\text { futuro médico }\end{array}$ & $\begin{array}{l}\text { Com hase na percep̧̧ào faworivel dos estudantes } \\
\text { pesquisados, é recomendivel a contibuidade do } \\
\text { julgamento simulado como uma das estratégias } \\
\text { de ensino da ética médica na instituição. }\end{array}$ \\
\hline 2014 & $\begin{array}{l}\text { Camargo et } \\
\text { al". }\end{array}$ & 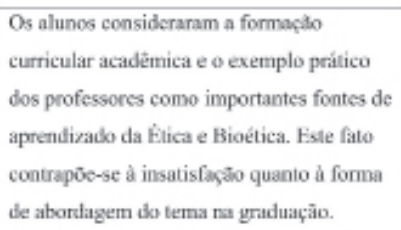 & $\begin{array}{l}\text { Conclui-se que se deve repensar o modelo de } \\
\text { ensino adotado para o tema ética médica e } \\
\text { biótica, em mazâo das criticns reoebidas. }\end{array}$ \\
\hline 2014 & Yambaki et al ${ }^{2}$. & 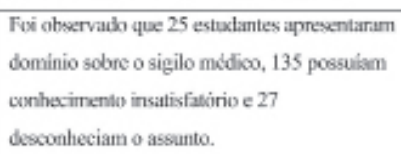 & 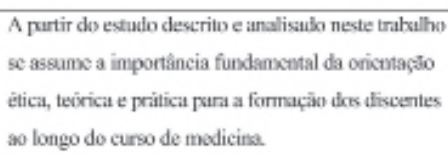 \\
\hline 2014 & Lisboe et al'... & $\begin{array}{l}\text { Foram identificados quatro códigns. Os } \\
\text { códigos sà̀ similares e embassdos pelo } \\
\text { Código de ftica Médica. }\end{array}$ & $\begin{array}{l}\text { Sugere-se ao Conselho Federal de Medicina a } \\
\text { elaboraçào de um código unificado que possa } \\
\text { contribuir de maneira significativa para a } \\
\text { educaçầo médica no Brasil. }\end{array}$ \\
\hline 2014 & Godoy et all ${ }^{11 . .}$ & $\begin{array}{l}\text { Houve nítida evoluçâo de conhecimento da } \\
\text { primeira para a quarta série, porém, a partir } \\
\text { da quarta séric, bouve estagnaçấo do } \\
\text { conhecimento. }\end{array}$ & $\begin{array}{l}\text { Há necessidade do ensino fommal da Ética Médica } \\
\text { đurante todos os anos da graduação, já que o } \\
\text { modelo atual, com ensino apenas na tereeira } \\
\text { ś́rie, se mostrou inelicaz. }\end{array}$ \\
\hline \multirow[t]{2}{*}{2017} & $\begin{array}{l}\text { Meneres et } \\
\text { al. }\end{array}$ & 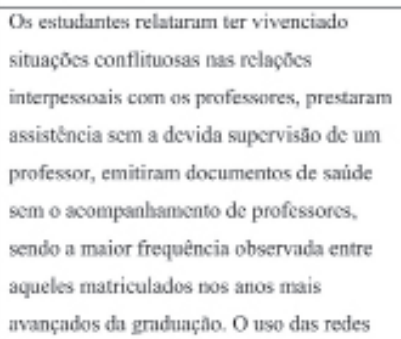 & $\begin{array}{l}\text { Faz-se necessario propiciar um espaç̧o de } \\
\text { discussão e de reflexăo coletiva acerca dos } \\
\text { problemas éticos vivenciadox pelos estudantes, ao } \\
\text { longo da graduaçăo em Modicina, a fim de se } \\
\text { construir um agir profissional eticamente cometo. }\end{array}$ \\
\hline & & $\begin{array}{l}\text { sociais com a finalidade de compartilhar } \alpha \\
\text { dxalos de pocientes foi prevalente nos anes } \\
\text { mais avançados da graduação. }\end{array}$ & \\
\hline 2013 & Bellbi ct $a^{b}$. & $\begin{array}{l}\text { A obra de Saramago desenvolvea bos } \\
\text { alunos as capacidades interpretativa, } \\
\text { reflexiva e critica }\end{array}$ & $\begin{array}{l}\text { A literatura, represcntada neste estudo pela leitura } \\
\text { do livro Ensaio sobre a cegueira, de José } \\
\text { Saramago, contribuiu de maneira positiva ná } \\
\text { aprendizagen dos principios éticos e bixéticos, } \\
\text { tendo acrescentado à formaçio pessoal e } \\
\text { profissional desses estudantes }\end{array}$ \\
\hline 2019 & $\begin{array}{l}\text { Menezes ef } \\
\text { al.s }\end{array}$ & $\begin{array}{l}\text { Os entrevistados relatam a desvalorizaplo e } \\
\text { dissociação do ensino de ética em relaç̃ó à } \\
\text { prítica, ressaltando a necessidade de } \\
\text { qualificaşāo dos docentes. }\end{array}$ & 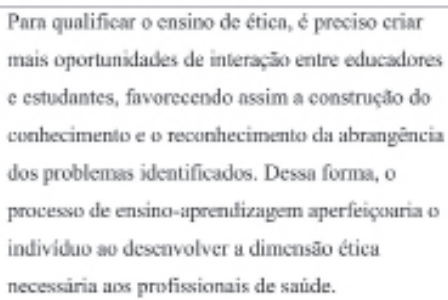 \\
\hline
\end{tabular}


Quadro 1. (cont.) Distribuição dos artigos conforme o ano de publicação, autoria, resultados e conclusões.

\begin{tabular}{|c|c|c|c|}
\hline 2019 & Gricas ef nl? & $\begin{array}{l}\text { Um terço dos discentes que nâo tivernm } \\
\text { contato com a temática revelaram não se } \\
\text { sentir prejudicndos por essa lacuna e } 25,0 \% \\
\text { da amustra sequer valorizou a impurtância } \\
\text { do tema em relaçio a outras disciplinas da } \\
\text { graduaçàto. }\end{array}$ & $\begin{array}{l}\text { Assim, torna-se necessário rever as estratégias do } \\
\text { ensino múdico para garantir melhores } \\
\text { profissionais no futuro. }\end{array}$ \\
\hline 2020 & Lima et al. ${ }^{12}$ & $\begin{array}{l}\text { A maionia des estudanies mustruu } \\
\text { conheximento suficiente, e o décimo primeiro } \\
\text { semestre obteve mais acertos novs casos" } \\
\text { problema propostos no questionário. }\end{array}$ & $\begin{array}{l}\text { Toma-:e necescánio aprofundar continuarnente o } \\
\text { estudo da temática de forma transversal, devido a } \\
\text { sua imņortincia na relaçầo médico-paciente. }\end{array}$ \\
\hline 2020 & Berbosa ct al $1^{9}$. & $\begin{array}{l}\text { A maior parte dos alunos afirmou já ter lido } \\
\text { o Código de Ếtica Médica e considerar sua } \\
\text { linguagem de fácil entendimento. Exceto } \\
\text { por pequenas variaçóes, o conhecimento } \\
\text { demonstrou-se progressivo so longo dos } \\
\text { semestres }\end{array}$ & $\begin{array}{l}\text { Os resultados confimsam a nccessidade de } \\
\text { aprofundamento constante sohre a ética médica, } \\
\text { pilar da formação e da prática. É preciso discutir } \\
\text { e refletir coletivamente sobre o tema, tomando } \\
\text { como base os entes dirctamente eavolvidos: } \\
\text { CFM, instituipốcs de ensino, docentes c } \\
\text { discentes. Só assim a ética fundamentará de fato a } \\
\text { atuaçâo profissional. }\end{array}$ \\
\hline 2020 & Teixeira et al $^{4}$ & 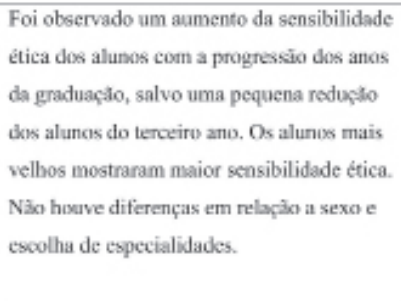 & 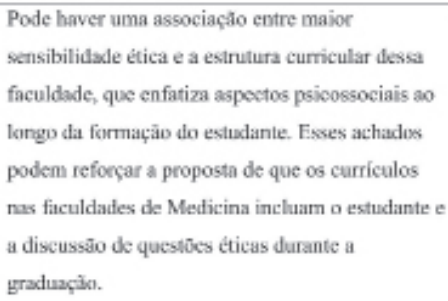 \\
\hline
\end{tabular}

Fonte: Dados do estudo, 2020.

disciplina e/ou ensino de ética médica.

Estudos revelam que na percepção dos estudantes de medicina existe uma desvalorização do ensino de ética médica na graduação, bem como a dissociação entre teoria e prática. Deixando claro que durante o curso a abordagem das humanidades é mínima, seu ensino é pontual e superficial, e que é imperativa a educação permanente em ética de professores e preceptores ${ }^{8}$.

O período em que se deve abordar a ética médica nas faculdades de medicina também foi alvo de discussão. Considera-se que o ensino de ética médica não pode ser pontual, é necessário que seja ensinado continuamente, durante e após a vida acadêmica, pois na área da saúde a capacidade do profissional de solucionar dilemas é testada com frequência, confirmando a necessidade de aprofundamento contínuo sobre a ética médica ${ }^{2,3,9}$.

Embora se tenham consciência da importância das aulas expositivas dialogadas ${ }^{10}$. Foi defendida a necessidade da existência de momentos de interatividade no ensino de ética na graduação de medicina, e de metodologias complementares onde o aluno possa participar efetivamente da sua organização e execução, isso facilitará a construção de conhecimentos e melhor compreensão dos problemas identificados no decorrer da graduação ${ }^{8,10}$.

Ouseja,háuma preocupação emintegrardiferentes abordagens para o ensino da ética médica. Ressaltado metodologias que estimulem os alunos à reflexão e ao pensamento crítico. Entre outras abordagens sugeridas e que foram identificadas como estratégias educacionais eficazes, destacam-se as discussões de casos, seminários, julgamento simulado, literatura, discussão em grupo, filmes e dramatização. Pois se considera que essas técnicas didáticas geralmente são estimuladoras para os alunos e para os resultados da aprendizagem como um todo $^{6,10}$.

No entendimento da literatura consultada ${ }^{6}$, a promoção da transdisciplinaridade e a utilização de metodologia pedagógica ativa sãoestratégiasimportantes que podem ser utilizadas para contextualizar o ensino da ética e da bioética no decorrer do curso de medicina: A transdisciplinaridade envolve a comunicação entre os docentes. Esse diálogo pode ocorrer por meio de oficinas pedagógicas como uma maneira de aproximar os saberes e facilitar o desenvolvimento da competência moral do futuro médico. Quanto ao emprego de metodologias pedagógicas ativas, acredita-se que possa também influenciar muito a formação moral do aluno, visto que permite a reflexão e o debate de ideias.

Três estudos tentaram avaliar o conhecimento e/ ou comportamento dos estudantes em relação ao sigilo na relação médico-paciente. Estudo realizado por Lima ${ }^{12}$ verificou que o conhecimento dos estudantes sobre o tema ainda é deficiente, mesmo em alunos em níveis mais avançados da graduação. Menezes e colaboradores ${ }^{7}$ mostrou a existência de compartilhamento de dados de 
pacientes nas redes sociais pelos alunos. Enquanto que, Yamaki $^{2}$ verificou que a maioria dos estudantes acredita ser eticamente aceitável comentar os casos vivenciados durante as atividades práticas.

Isso implica afirmar que os dilemas éticos na medicina surgem muito cedo, começando antes mesmo da atuação profissional do médico. A transgressão do Código de Ética Médica, especificamente das normas deontológicas, pode levar o infrator a receber penas legais ${ }^{11-32}$. Logo, o conhecimento dos alunos onde o sigilo pode ou não ser quebrado é de grande importância, havendo consenso de que a conduta ética precisa começar a ser construída na formação inicial do estudante, a fim de se construir um agir profissional eticamente correto $^{2,7,12}$.

\section{Conclusão}

Verificou-se que a maioria dos artigos sobre o ensino da ética na graduação de medicina procura apresentar propostas de ensino que possam facilitar o ensino e a aprendizagem da disciplina e propiciar a formação de um profissional reflexivo e crítico.

É consenso entre os pesquisadores de que o ensino de ética médica precisa ser desenvolvido durante toda a graduação e que a interdisciplinaridade e fundamental, uma vez que pode facilitar a compreensão do aluno sobre os princípios éticos e da sua importância para a profissão médica, ao contribuir para o enfrentamento de dilemas éticos no durante a prática profissional.

Conclui-se que existem deficiências no ensino de ética médica, mas essas podem ser superadas quando houver a valorização da disciplina nos cursos de graduação de medicina e, sobretudo, nas universidades brasileiras.

\section{Referências}

1. Lisboa L, Lins L. Código de ética do estudante de medicina: uma análise qualitativa. Rev. Bioét. 2014;22(1):182-190.

2. Yamaki VN, Teixeira RKC, Oliveira JPS, Yasojima EY, Silva JAC. Sigilo e confidencialidade na relação médico-paciente: conhecimento e opinião ética do estudante de medicina. Rev. Bioét. 2014 Apr; 22(1):176181.

3. Graças VBA, Souza Júnior JF, Santos JGMS, Almeida MFA, Oliveira EVG, Santos NVMO. Conhecimento sobre ética médica e resolução de conflitos na graduação Rev. bioét. Out.-Dec. 2019; 27(4): 643-660.

4. Teixeira RS, Silva RL, Silva LF, Miranda TLKS, Teixeira RKC, Silva JAC. Bioética e discentes de medicina: transição do método de ensino Rev. bioét. 2019; 27(1):127-133.

5. Camargo A, Almeida MAS, Morita I. Ética e bioética: o que os alunos do sexto ano médico têm a dizer. Rev. bras. educ. med. 2014 June; $38(2): 182-189$.

6. Balbi L, Lins L, Menezes MS. A Literatura como Estratégia para Reflexões sobre Humanismo e Ética no Curso Médico: um Estudo Qualitativo. Rev. bras. educ. med. 2017; 41(1):152-161.
7. Menezes MM, Maia LC, Rocha CU, Sampaio CA, Costa SM. Conflitos Éticos Vivenciados por Estudantes de Medicina. Rev. bras. educ. méd. Jan.-Mar. 2017; 41(1):162-169.

8. Menezes MM, Maia LC, Abreu MHNG, Sampaio CA, Costa SM. Percepções sobre o ensino de ética na medicina: estudo qualitativo Rev. bioét. 2019; 27(2):341-349

9. Barbosa JS, Silva PD, Neves NMBC. Conhecimento em ética médica entre estudantes de medicina de Salvador. Rev. Bioét. 2020; 28(2):307-318. 10. Savaris PK, Reberte A, Bortoluzzi MC, Schlemper JB, Bonamigo EL. Julgamento simulado como estratégia de ensino da ética médica. Rev. Bioét. 2013 Apr; 21(1):150-157.

11. Godoy MF, Ferreira HRA, Pria OAFD. Avaliação do conhecimento da ética médica dos graduandos de medicina. Rev. bras. educ. med.. 2014; 38(1), 31-37.

12. Lima SMFS, Silva SMM, Neves NMBC, Crisostomo LML. Avaliação do conhecimento de estudantes de medicina sobre sigilo médico. Rev. bioét 2020; 28(1):98-110. 